J. Clin. Chem. Clin. Biochem.

Vol. 14, 1976, pp. 83-92

\title{
Über Kathepsin, Phosphoproteid-Phosphatase und Saure Phosphatase in der löslichen Fraktion aus Rinderhirn-Cortex; Reinigung und Eigenschaften ${ }^{1}$ )
}

\author{
Von Elfriede Albert \\ Aus dem Biochemischen Institut der Universität Uppsala/Schweden und dem Physiologisch-chemischen Institut \\ (Lehrstuhl II) der Universität zu Köln
}

(Eingegangen am 1. April/1. Juli/27. Oktober 1975)

Herrn Professor Dr. Karl Leonhard zum 70. Geburtstag gewidmet

Zusammenfassung: Aus der Großhirnrinde des Rindes wurde die lösliche Fraktion in 0,29 mol/1 Saccharoselösung bereitet. Daraus konnten durch Separation auf Sephadex G 200 in neutralem Puffer drei am Proteinumsatz beteiligte Enzyme abgetrennt werden: Kathepsin (EC 3.4.4.23) Phosphoproteid-Phosphatase (EC 3.1.3.16) und Saure Phosphatase (EC 3.1 3.2). Das Kathepsin wurde durch Gelfiltration auf Sephadex G 100 und Säulenelektrophorese bis 380 -fach gereinigt. Das pH-Optimum lag um pH 5,7. Bei $37^{\circ} \mathrm{C}$ trat binnen 30 min keine Aktivitätsabnahme ein. Die $K_{\mathrm{m}}$ betrug 2,75 mg/ml Casein Hammarsten. Das Molekulargewicht des Kathepsins aus dem Elutionsvolumen nach Gelfiltration und Exclusions-Gel-Elektrophorese lag bei 45000 , was dem des Kathepsins aus menschlicher Leber entspricht (Barrett, A. J. (1970) Biochem. J. 117, 601-607). Die Sedimentationskonstante 3,9 $\mathrm{s}_{20, \mathrm{w}}$ ist vergleichbar mit den Größen von Proteinasen anderer Herkunft, ebenso der Aufbau aus vorwiegend sauren Aminosäuren. Für die Phosphoproteid-Phosphatase und Saure Phosphatase wurden weitere Reinigungsmöglichkeiten auf Säulenelektrophorese und Hydroxylapatit aufgezeigt. Die Phosphoproteid-Phosphatase war gekennzeichnet durch ein $\mathrm{pH}-$ Optimum um pH 5,5. Bei $45^{\circ} \mathrm{C}$ war binnen 20 min keine Aktivitätsabnahme meßbar. Die $K_{\mathrm{m}}$ betrug $1,43 \mathrm{mg} / \mathrm{ml}$ Casein isoelectric. Die Saure Phosphatase war ausgezeichnet durch ein pH-Optimum von 5,6 . Bei $54^{\circ} \mathrm{C}$ war binnen 30 min keine Aktivitätsabnahme zu messen. Die $K_{m}$ für Na-Phenolphthaleindiphosphat betrug $2 \mu \mathrm{mol} / 1$. Alle drei Enzyme verloren langsam nach einigen Wochen bei $-4^{\circ} \mathrm{C}$ ihre Aktivität, offenbar durch Selbstverdauung in der Kälte.

\section{Cathepsin, phosphoprotein-phosphatase and acid phosphatase in the soluble fraction of the cattle brain cortex: purification and properties}

Summary: Cattle brain cortex was homogenised in 0,29 mol/l sucrose and centrifuged at $101000 \times \mathrm{g}$. The supernatant contains the majority of 3 enzymes participating in protein turnover: cathepsin (EC 3.4.4.23), phosphoprotein phosphatase (EC 3.1.3.16) and acid phosphatase (EC 3.1.3.2). They were separated by chromatography on Sephadex G 200 in neutral buffer. The cathepsin was purified up to 380 fold by gel filtration on Sephadex and column electrophoresis. The $\mathrm{pH}$ optimum of cathepsin was 5.7 . At $37^{\circ} \mathrm{C}$ no decrease of activity was measurable during $30 \mathrm{~min}$. The $\mathrm{K}_{\mathrm{m}}$ was found to be $2.75 \mathrm{mg} / \mathrm{ml}$ Casein Hammarsten. The molecular weight by gel filtration and exclusion-gel electrophoresis was about 45000 , corresponding to the cathepsin from human liver (Barrett, A. J. (1970) Biochem. J. 117, 601-607). The sedimentation constant $3.9 \mathrm{~s}_{20, \mathrm{w}}$ is comparable with the values of proteinase of different origin, and the composition is similar with respect to the high proportion of acidic amino acids. The phosphoprotein phosphatase can be further purified by chromatography on hydroxyapatite and by column electrophoresis. The $\mathrm{pH}$ optimum of phosphoprotein phosphatase was about $\mathrm{pH} 5.5$. At $45^{\circ} \mathrm{C}$ no decrease of activity was measurable during $20 \mathrm{~min}$; the $K_{\mathrm{m}}$ was $1.43 \mathrm{mg} / \mathrm{ml}$ casein isoelectric. The $\mathrm{pH}$ optimum of acid phosphatase was about 5.6. At $54^{\circ} \mathrm{C}$ no decrease of activity was measurable during $30 \mathrm{~min}$; the $K_{\mathrm{m}}$ was $2 \mu \mathrm{mol} / 1$ for Sodium phenolphthalein diphosphate. All three enzymes slowly lost their activity during several weeks at $-4^{\circ} \mathrm{C}$, apparently by self digestion in the cold.

1) Gefördert durch Mittel der Deutschen Forschungsgemeinschaft. 


\section{Einleitung}

Die intrazellulären Proteinasen ${ }^{2}$ ) sind in den meisten Zellen vorhanden. Sie wirken mit am Abbau und Umbau von Zell- und Strukturproteinen $(2,3,4,5)$, sowie an der Aktivierung oder Zerstörung von Enzymen $(6,7)$. Mehrere proteolytische Enzyme finden sich in den Lysosomen von Leber und Milz als membranumschlossene Funktionseinheiten angeordnet, was den Abbau der Proteine in konzertierter Aktion ermöglichen soll, die unter Wechsel des pH in immer kleinere Bruchstücke zerlegt werden $(8,9)$. Kathepsin E ( $\mathrm{pH} \mathrm{2,5)} \mathrm{und} \mathrm{Kathepsin} \mathrm{D}$ $(\mathrm{pH} 3,8)$ greifen große Proteinmoleküle an, während die Kathepsine A, B und C, die im schwach sauren Bereich wirken ( $\mathrm{pH} 5,3)$ eigentliche Peptidasen sind $(1,2,9,10$, $11,12)$, wobei aber Kathepsin $C$ auch Transamidierungen katalysieren (13) und Kathepsin B auch einige Eiweißkörper aufspalten kann (14). Außer den eigentlichen Proteinasen finden sich in den Lysosomen auch die Phosphoproteid-Phosphatase (pH unter 5,8) (15) und die Saure Phosphatase (15). Letztere Aktivität dürfte ebenfalls mit dem Eiweißumsätz verknüpft sein, da sie in Zellen mit gesteigerter Proteinsynthese vermehrt vorhanden ist.

Aus Bakterien und Hefe wurden saure Proteinasen isoliert vom Molekulargewicht 32 000-38 000 und einer Sedimentationskonstanten von $3,35 s_{20, w}(16,17)$. In ihrer Aminosäure-Zusammensetzung zeichneten sie sich, ähnlich wie Pepsin, durch hohen Gehalt an sauren Aminosäuren, Hydroxy-Aminosäuren und Prolin und extrem niedrigen Gehalt an basischen Aminosäuren, Tryptophan und schwefelhaltigen Aminosäuren $(17,18)$ aus. Die meisten Proteinasen von Säugern, Streptokokken, Aspergillus und die Proteinasen B und $\mathrm{C}$ aus Hefezellen liegen in einer inaktiven Proform als Zymogen vor (16, 19a, 19b). In der Hefe sind diese aktivierbar unter Abspaltung eines Hemmproteins durch saures $\mathrm{pH}$ ( $\mathrm{pH} 5)$ oder denaturierende Mittel wie Harnstoff, Aceton und gewisse Alkohole.

Im Säugerhirn sind 3 Kathepsine beschrieben von saurem (pH 3,5-3,8), schwach saurem ( $\mathrm{pH} \mathrm{5,4)} \mathrm{und} \mathrm{neutralem} \mathrm{-}$ (pH 7,7-8,0) Wirkungsoptimum, außerdem eine Phosphoproteid-Phosphatase, die im schwach sauren Bereich ( $\mathrm{pH} \mathrm{5,5)} \mathrm{wirkt.} \mathrm{Allen} \mathrm{ist} \mathrm{gemeinsam,} \mathrm{daß} \mathrm{sie} \mathrm{von} \mathrm{zahl-}$ reichen angebotenen Substraten wie Dipeptiden, Modellpeptiden, löslichen Hirnproteinen und physiologisch wichtigen Phosphatverbindungen nur selten eines in geringem Ausmaß angriffen, aber regelmäßig Phosphoproteide, meistens geprüft an Casein, aufgespalten haben $(6,20)$. Im Hirngewebe kommt Casein zwar nicht vor,

\footnotetext{
${ }^{2}$ ) Der Trivialname Saure Phosphatase wird hier gebraucht für das Enzym Orthophosphoric monoester phosphohydrolase EC 3.1.3.2, Phosphoproteid-Phosphatase für Phosphoprotein phosphohydrolase EC 3.1.3.16 und Kathepsin für Peptide peptidohydrolase EC 3.4.4.23.

Enzymaktivitäten werden in $\mathrm{mmol} / \mathrm{min}$ Tyrosinäquivalente bzw. nmol/min Phosphat angegeben. Spezifische Aktivitäten sind auf $\mathrm{mg}$ Protein bezogen.
}

aber native Phosphoproteide vom gleichen Polyphosphoryl-Seryltyp wie Casein, die altemierend phosphoryliert und dephosphoryliert werden $(21,22,23)$ und mit dem Kationentransport unter der Erregungsleitung verknüpft gedacht werden $(5,21,22)$. Die saure Proteinase wurde wie die neutrale Proteinase in der löslichen Fraktion aufgefunden $(6,24,25,26)$, die im schwach sauren Bereich wirksame, sehr aktive Proteinase in den Lysosomen (27). Die neutrale Proteinàse, von Guroff (6) aus der löslichen Fraktion von Rattenhirn 50-100 fach gereinigt, war $\mathrm{Ca}^{++}$abhängig, spaltete linear der Zeit für $40 \mathrm{~min}$, wurde gehemmt durch höhere Caseinkonzentrationen und war über $30^{\circ} \mathrm{C}$ labil. Die PhosphoproteidPhosphatase aus Rinderhirn wurde 100-fach gereinigt (20), fand sich zu 60\% im Cytoplasma, war aktivierbar durch $\mathrm{Mg}^{++}$und $\mathrm{Mn}^{++}$, empfindlich gegen Ammoniumsulfat und wurde durch höhere Substratkonzentrationen von Casein gehemmt.

In früheren Untersuchungen (28) haben wir die einzelnen Partikelfraktionen und den Uberstand aus Rinderhirnhomogenat auf ihre Aktivität gegen Na-Phenolphthaleindiphosphat (pH 5,6 und 9,7) geprüft als Maß für die saure und die alkalische Phosphatase. Je nach Hirnregion fand sich bis $\mathrm{zu} 61 \%$ der Aktivität des Homogenats an saurer Phosphatase im partikelfreien Ửberstand, während sich die alkalische Phosphatase vorwiegend in den Mikrosomen fand. Die Fähigkeit der Mikrosomen und der löslichen Fraktion, organische Phosphatverbindungen anzugreifen, wurde gegenüber 14 biologisch wichtigen Phosphatverbindungen geprüft. Das einzige hochmolekulare Substrat, Casein, wurde als einzige Substanz von der löslichen Fraktion aufgespalten. In späteren Untersuchungen (29) an Ratten- und Mäusehirnen wurde direkt von der löslichen Fraktion ausgegangen und geprüft, ob die Casein-spaltende Aktivität an den Peptidbindungen oder an den Phosphatbindungen angreift. Es fanden sich beide Aktivitäten nebeneinander im Sinne einer Proteinase (pH 5,4) und einer Phoșphoproteid-Phosphatase (pH 5,6). Die Enzyme entwickelten sich im Mäusehirn unabhängig voneinander je nach Lokalisation und Reifegrad des Gewebes.

In den nachfolgend dargestellten Untersuchungen wurde versucht, diese von uns früher in der löslichen Fraktion des Säugerhirns aufgefundenen Enzyme zu reinigen und zu charakterisieren.

\section{Material und Methoden}

Bereitụng von Homogenat und löslicher Fraktion 109 Rinderhịne wurden insgesamt verarbeitet in 26 einzelnen Separationsperioden. Die Großhirnrinde wurde in flachem Tangentialschnitt abgetragen. Es wurden Feuchtgewichte zwischen 90 und $760 \mathrm{~g}$ homogenisiert. Von 5 Rinderhirnen wurden im Mittel $660 \mathrm{~g}$ Feuchtgewicht an Rindengewebe gewonnen und mit sterilisierter 0,29 mol/1 Saccharoselösung mit einem Porzellanpistill durch ein feinès Drahtsieb gestrichen. Diese Suspension wurde homogenisiert in einem Glashomogenisator nach PotterElvehjem mit Teflonpistill und koliert durch döppelten Mull. Das Homögennat, im Mittel $1250 \mathrm{ml}$, wurde zuerst bei niedrigerer Geschwindigkeit von groben Partikeln befreit ( $30 \mathrm{~min} 34000 \times g$, Servall) und dạnn in der Spinco-Ultrazentrifuge, Modell L, mit 
Rotor 40,2 bei $101000 \times g$ in 60 min die feineren Partikel entfernt. Der Überstand wurde als lösliche Fraktion und Enzympräparat benützt $(\overline{\mathrm{x}} 620 \mathrm{ml})$. Alle Glasgefäße, Plastikgeräte und Lösungen waren zuvor sterilisiert worden. Den Puffern wurde als Bakterizid $3 \mathrm{mmol} / 1 \mathrm{NaN}_{3}$ zugesetzt, das in der verwendeten Konzentration die Enzyme nicht hemmte. Alle Arbeitsgänge wurden im Kühlraum bei $+4^{\circ} \mathrm{C}$ durchgeführt. Die benützten Zentrifugen waren automatisch gekühlt.

\section{Konzentricrung der löslichen Gewebsproteine}

Die Proteinlösungen wurden durch Ultrafiltration in Dialyseschläuchen (Visking) im Vakuum konzentriert. Andere Konzentrierungsmethoden brachten nicht den gewünschten Erfolg. Nach Konzentrierung der in Dialyseschläuchen befindlichen Proteinlösung in fester Saccharose wurden zwar die Enzyme hochaktiv bewahrt, doch blieben inaktive Proteine störend beigemischt bis in späte Reinigungsschritte. Konzentrierung durch Fällung mit Ammoniumsulfat (25-60\% Sättigung) beeinträchtigte die Enzymaktivität, am stärksten die der Sauren Phosphatase, aber auch die der beiden Proteinasen.

\section{Auftrennung der Proteine}

\section{Gelfiltration}

a) Sephadex (Pharmacia, Uppsala/Schweden) G 200: Säule $3,4 \times 160 \mathrm{~cm}$, Vo $580 \mathrm{ml}$, Vt $1760 \mathrm{ml}$, Vi $1180 \mathrm{ml}$, äquilibriert mit $0,02 \mathrm{~mol} / 1$ Tris-HCl, $\mathrm{pH} 7,2,0,1 \mathrm{~mol} / 1$ an $\mathrm{NaCl}$. Die Lösung des Proteins wurde von unten in die Säule eingebracht. Die Elutionsgeschwindigkeit lag bei $25 \mathrm{ml} / \mathrm{h}$, LKB Pumpe.

b) Sephadex G 100: Säule $3,2 \times 220 \mathrm{~cm}$, Vo $500 \mathrm{ml}$, Vt $1710 \mathrm{ml}$, Vi $1210 \mathrm{ml}$, äquilibriert mit $0,02 \mathrm{~mol} / \mathrm{l}$ Tris- $\mathrm{HCl}, \mathrm{pH} 7,20,1 \mathrm{~mol} / \mathrm{l}$ $\left(\mathrm{NH}_{4}\right)_{2} \mathrm{SO}_{4}$. Die meisten Kathepsinpräparate wurden nur einmal separiert, manche aber mehrmals bis zu 8 maliger Rezyklisierung. Es wurde auch auf dünneren Säulen $(2 \times 200 \mathrm{~cm})$ mit Sephadex $G 100$ und $G 75$ separiert, um schärfere Gipfel zu erhalten.

\section{Elektrophorese}

Erste Versuche, Kathepsin auf Cellulose (Schleicher und Schüll) als Träger zu separieren, mußten aufgegeben werden wegen Inaktivierung des Enzyms. Als Träger wurde Sephadex G 25-Gel verwendet in Säulen $1 \times 50$ und $2 \times 50 \mathrm{~cm}$, äquilibriert mit 20-50 mmol/1 Tris-HCl-Puffer, pH 7,2-8,5, später mit $20 \mathrm{mmol} / 1$ Natriumphosphatpuffer von pH $5,7 / 6,0 / 6,2$ bis 6,5 wegen etwas besserer Beweglichkeit des Kathepsinproteins im schwach sauren Milieu. Phosphoproteid-Phosphatase und Saure Phosphatase wurden in Tris- $\mathrm{HCl}$ Puffer $\mathrm{pH} 7,2,20 \mathrm{mmol} / 1$ separiert. Elektrisches Feld 400 bis 1000 V, 15-50 mA, 7-77 Stunden. Die Elutionsgeschwindigkeit lag zwischen 2,2 und $8,5 \mathrm{ml} / \mathrm{h}$.

\section{Hydroxylapatit-Gel}

Die Säule, gefüllt mit Hydroxylapatit nach Tiselius (33) war äquilibriert mit 0,01 mol/1 Kaliumphosphatpuffer, $\mathrm{pH}$ 7.0. Bei jedem der drei Enzyme wurde zunächst schrittweise eluiert mit 0,02/0,04/0,06/0,08/0,10/0,15/0,4/0,5/1.0 mol/1 Kaliumphosphatpuffer pH 7,0. Die Phosphoproteid-Phosphatase eluierte bereits mit $10 \mathrm{mmol} / 1$ Kaliumphosphatpuffer in einer Front inaktiven Proteins. Hier war die Bestimmung der phosphatabspaltenden Aktivität erst möglich nach Wiederabtrennung des Phosphatpuffers von der Proteinfraktion auf Sephadex G 25. Kathepsin und Saure Phosphatase eluierten mit $60 \mathrm{mmol} / 1 \mathrm{Kaliumphos-}$ phatpuffer.

\section{Ionenaustäuscher}

Die mit CM-Cellulose (Serva, Heidelberg) gefüllte Säule war äquilibriert mit Na-Phosphatpuffer $\mathrm{pH} 6,5,10 \mathrm{mmol} / 1$. Es wurde eluiert mit $0,01 / 0,02 / 0,05 / 0,10 / 0,5 \mathrm{~mol} / 1$ Na-Phosphatpuffer $\mathrm{pH}$ 6,5 . Kathepsin trat bereits mit $10 \mathrm{mmol} / 1 \mathrm{Na}$-Phosphatpuffer aus.

DEAE-Celluilose (Serva, Heidelberg):

Aus der mit $50 \mathrm{mmol} / 1$ Tris-HCl Puffer $\mathrm{pH} 8,0$ äquilibrierten Săule eluierte das Kathepsin-Protein nach Einrichtung eines $\mathrm{NaCl}-$ Gradienten $(0,05 \mathrm{~mol} / \mathrm{l}$ Tris- $\mathrm{HCl}$ gegen $1,0 \mathrm{~mol} / \mathrm{A} \mathrm{NaCl}$ im gleichen Puffer) mit 0,04 mol/1 NaCl. Die PhosphoproteidPhosphiatase ließ sich mit 20 mmol/1 Tris-HCl, pH 7,2, 5 mmol/1 an $\mathrm{NaCl}$ nicht von der Säule lösen. Das gelang erst nạch Ạuilibrieren der Säule mit $2 \mathrm{mmol} / 1$ Acetatpuffer $\mathrm{pH} \mathrm{6,0} \mathrm{und}$ Einrichtung eines Gradienten gegen $1,0 \mathrm{~mol} / 1 \mathrm{NaCl}$ im gleichen Puffer (nach H. R. Revel).

\section{Agar-Gel-Elektrophorese}

Das Agarose-Gel in 0,05 mol/1 Tris-Acetatpuffer pH 7,2, war freundlichst von Prof. Stellan Hjertén, Uppsala, zur Verfügung gestellt worden. Die Wanderung der Kathepsinfraktion wurde nach $23 \mathrm{~h}(5 \mathrm{~mA}, 800 \mathrm{~V})$ geprüft. Dazu wurden gemäß der Stricheinteilung fortlaufend Proben aus der Gclsäulc entnommen, das enthaltene Gel abzentrifugiert und im Überstand die Aktivität bestimmt. Die aktiven Fraktionen wurden vereinigt, das Protein rekonzentriert und auf Sephadex G 75 in Tris-HCl Puffer scpariert, um Gelreste zu entfernen, die die anschließende Aufnahme der Proteinkurve bei 280 nm störten.

\section{Bestimmung der Enzymaktivität}

\section{Saure Phosphatase}

Die Aktivität wurde bestimmt an der Aufspaltung des Substrates Phenolphthalein-diphosphat (Serva, Heidelberg). Die Farbintensität von freigesetztem Phenolph thalein wurde in alkalischem Milieu bei $540 \mathrm{~nm}$ gemessen. Die Methode von Huggins \& Talalay (34) wurde für das Inkubationsvolumen von $0,8 \mathrm{ml}$ modifiziert. Der Inkubationsansatz enthielt $0,1 \mathrm{ml}$ Eluat. Als Phosphatase-Einheit gilt das kolorimetrische Äquivalent für $1 \mu \mathrm{g}$ Phenolphthalein, freigesetzt unter Inkubationen von $60 \mathrm{~min}$ bei $38^{\circ} \mathrm{C}$ und $\mathrm{pH} 5,6$. Meßvolumen $1,8 \mathrm{ml}$.

\section{Phosphoproteid-Phosphatase}

Substrat $50 \mathrm{~g} / 1$ Lösung von Casein isoelectric (Difco Laboratories, Detroit/USA, P-Gehalt $8,2 \mathrm{~g} / \mathrm{l}$ ) wurde durch Lösen unter Alkalizusatz (pH 6,9) hergestellt. Hieraus wurde eine 20 g/l Caseinlösung von pH 5,4 bereitet, indem $10 \mathrm{ml}$ der $50 \mathrm{~g} / \mathrm{l}$-Stammlösung mit Acetat-Veronalpuffer pH 4,6 nach Michaelis (35) auf $25 \mathrm{ml}$ aufgefullt wurden. Der Inkubationsansatz von $1,0 \mathrm{ml}$ enthielt bis zu $0,5 \mathrm{ml}$ des Eluates (Enzym) und $0,4 \mathrm{ml}(8 \mathrm{mg})$ Casein isoelectric, ad 1,0 mit $0,1 \mathrm{~mol} / 1$ Acetatpuffer $\mathrm{pH} 5,2$ und wurde 20-30 min inkubiert. Im säurelöslichen Überstand nach Trichloressigsäurefällung wurde das anorganische Phosphat nach Kuttner \& Lichtenstein (36) bestimmt und die Extinktion abgelesen bei $700 \mathrm{~nm}$. Die aus Enzym bzw. Substrat einzeln während der Inkubation freigesetzte Phosphatmenge wurde in Leerwerten ermittelt. Ebenso wurden Proben von P-Standard im gleichen Volumen des Puffergemisches parallel bestimmt.

\section{Kathepsin}

Das Kathepsin wurde nach Duspiva (37) bestimmt. Der Ansatz von $0,3 \mathrm{ml}$ enthielt $0,1 \mathrm{ml}$ einer $30 \mathrm{~g} / 1$ Lösung von Casein nach Hammarsten (Merck) in $8 \mathrm{~mol} / \mathrm{l}$ Harnstofflösung und wurde 30-60 min inkubiert. Das säurelösliche, Tyrosin-positive Material wurde im Überstand nach Trichloressigsäurefällung durch UVAbsorption bei $280 \mathrm{~nm}$ in $0,5 \mathrm{~cm}$ Quarzküvetten bestimmt. In Leerwerten wurde die Menge Tyrosin-positiven Materials bestimmt, das während der Inkubation aus dem Enzym bzw. dem Substrat freigesetzt wurde. Proben von Tyrosin-Standard wurden parallel nach derselben Methode bestimmt. Wegen der Empfindlichkeit des Nachweises gegenüber Detergentien mußten die Röhrchen für jeden Versuch mit Chromschwefelsäure gereinigt werden.

\section{Proteinbestimmung}

Protein wurde mit der Folin-Reaktion bestimmt in der Modifikation nach Lowry et al (38) mit kristallinem Rinder-Serumalbumin (Behring Werke Marburg) als Standard.

\section{Ergebnisse}

\section{Reinigung der Enzyme}

Die Konzentrierung der löslichen Gewebsproteine durch Ultrafiltration erbrachte unter mehreren Methoden die höchste Enzymausbeute. Gleichzeitig fiel dabei auch ein Teil der störenden hochmolekularen Proteine, die in der Gelfiltration mit der ersten Proteinfront eluieren, schon vorweg aus. 
Auch der Verdünnungsgrad der löslichen Fraktion war von Einfluß auf den Ertrag an Enzymaktivität. Das Verhältnis Hirnfeuchtgewicht zum Volumen der löslichen Fraktion lag in den verschiedenen Präparaten zwischen $1: 0,8$ und 1:3. Alle drei Enzyme waren in den verdünnteren Präparaten stärker aktiv.

Die Auftrennung der drei Enzymproteine erfolgte durch Gelchromatographie auf Sephadex G 200 (Abb. 1). Die drei aktiven Fraktionen wurden einzeln weiteren Reinigungsschritten unterworfen:

Die Kathepsinfraktion ergab auf Sephadex G 100 einen breiten, zweigipfligen Proteinpeak, in dessen Rückflanke die Enzymaktivität lokalisiert war (Abb. 2). Bei einigen Präparaten wurde versucht, durch 6-8 malige Rezyklisierung auf Sephadex G 100 Begleitproteine abzutrennen, was nicht gelang.

Bei der anschließend durchgeführten Elektrophorese (Abb. 3) wanderten die meisten der begleitenden Proteine zur Anode. Das Kathepsin bewegte sich im pHBereich zwischen 6,2 und 8,0 nur ganz gering in Richtung zur Kathode, zwischen pH 5,7 und 6,0 etwas rascher, ohne daß es sich ganz aus der Vorderflanke des Proteingipfels abgelöst hätte. Eine Abspaltung kam einzig zustande nach wiederholten mechanischen Einwirkungen auf das Protein unter den Konzentrierungsschritten (Abb. 4). Unter der Elektrophorese gelang es, die durch alle Gel-Passagen unverändert dem Kathepsin anhaftende rotgefärbte Proteinfraktion abzutrennen, da sie rascher zur Kathode wanderte. Der rote Farbstoff absorbierte maximal bei $420 \mathrm{~nm}$.

Weniger geeignet zur Reinigung erwiesen sich Ionenaustausch- und Adsorptionsverfahren (CM-Cellulose, DEAECellulose, Hydroxylapatit) wegen hoher Aktivitätsverluste.

Zur weiteren Reinigung der Phosphoproteid-Phosphatase erschien die Elektrophorese günstig. Die Saure Phosphatase ließ sich weiter reinigen auf Hydroxylapatit, von dem sie in $60 \mathrm{mmol} / 1$ Kaliumphosphatpuffer eluierte mit $75 \%$ Ausbeute, während sie unter Elektrophorese zwar Begleitproteine, aber auch stark an Aktivität verlor.
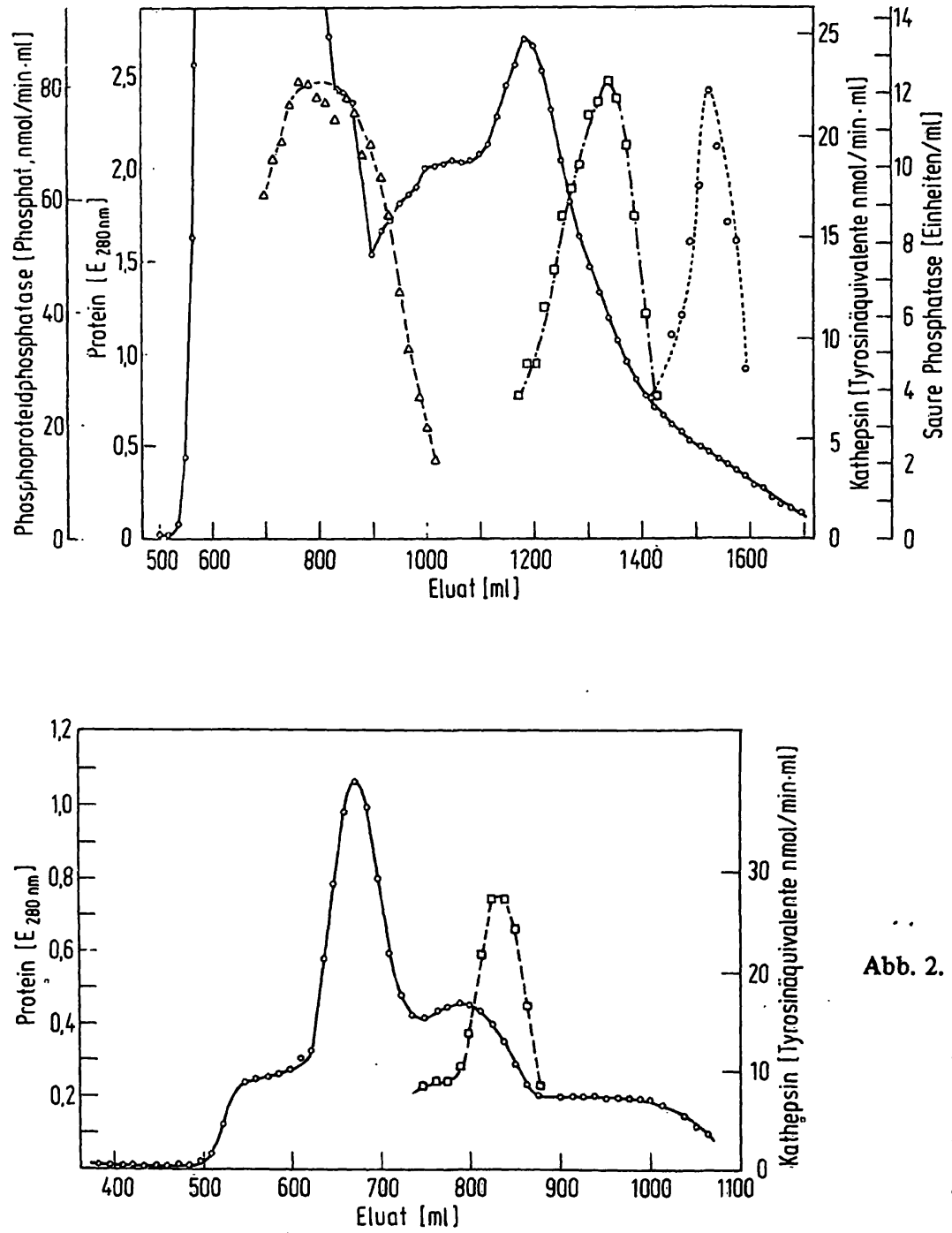

Abb. 1. Trennung auf Sephadex G 200. Lösliche Fraktion, $645 \mathrm{ml}$, Sediment nach Ammoniumsulfatzugabe $600 \mathrm{~g} / \mathrm{l}$, resuspendiert in $114 \mathrm{ml}$ $0,02 \mathrm{~mol} / 1$ Tris- $\mathrm{HCl}, 0,1 \mathrm{~mol} / 1$ an $\mathrm{NaCl}, \mathrm{pH} 7,2$. 2,78 g Protein. Von unten in die Säule $(3,4 \times 160 \mathrm{~cm})$, mit dem gleichen Puffer äquilibriert, aufgetragen. Elution $25 \mathrm{ml} / \mathrm{h}$. $\circ$ Protein $\Delta-\Delta-$ Phosphoproteidphosphatase 口- --- Kathepsin 0----o Saure Phosphatase
Abb. 2. Separation auf Sephadex G 100

Kathepsinfraktion aus Sephadex G 200 (s. Abb. 1), durch Ultrafiltration eingeengt auf $40 \mathrm{ml}$. Auftrag von unten auf die Säule $(3,2 \times 220 \mathrm{~cm})$, gefüllt mit Sephadex-Gel G 100, äquilibriert mit $0,02 \mathrm{~mol} / 1$ Tris- $\mathrm{HCl}, 0,1 \mathrm{~mol} / 1$ an $\left(\mathrm{NH}_{4}\right)_{2} \mathrm{SO}_{4}, \mathrm{pH} 7,2$. Elution $19 \mathrm{ml} / \mathrm{h}, \mathrm{LKB}$ Pumpe. $\circ \longrightarrow$ Protein - $\square$ Kathepsin 

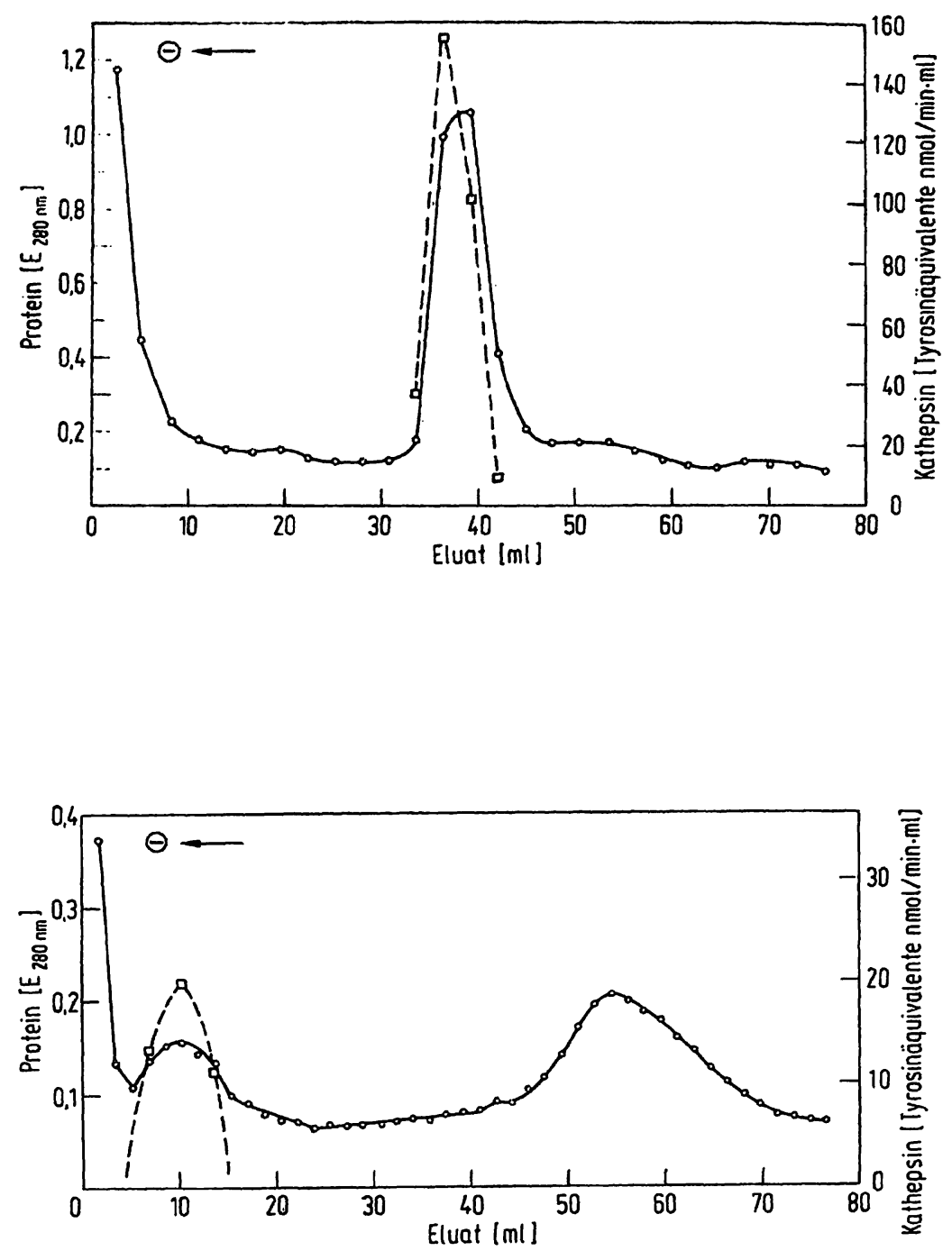

Abb. 3. Elektrophorese der Kathepsinfraktion. Kathepsinfraktion nach Passage von Sephadex G 200, 3 maliger RezykJisierung auf Sephadex G 100 (Abb. 2), 3maliger Elektrophorese auf Sephadex G 25 als Träger in $0,02 \mathrm{~mol} / \mathrm{l}$ Natriumphosphatpuffer $\mathrm{pH} 6,0$, Säule $2 \times 50 \mathrm{~cm}, 35 \mathrm{~mA}, 1300 \mathrm{~V}$, 54 Stunden $-\rightarrow \mathrm{K}^{-}$. Elution $8,4 \mathrm{ml} / \mathrm{h}$. Fraktion $20 \mathrm{~min}$. Nach allen Separierungsschritten Präparat durch Ultrafiltration eingeengt. Spezifische Aktivität (Tyrosinäquivalente) $162 \mathrm{nmol} / \mathrm{min} \cdot \mathrm{mg}$ Protein. 0 - 0 Protein $0-\cdots$ Kathepsin
Abb. 4. Aufspaltung einer bereits zweimal elektrophoretisch gereinigten Kathepsinfraktion bei 3. Elektrophorese unter Absinken der Aktivität auf 23\% und Erhöhung der Wanderungsgeschwindigkeit in Richtung auf die Kathode. Ein höherer, inaktiver Proteingipfel ist entstanden und in Richtung der Anode gewandert. Kathepsinfraktion nach Passage von Sephadex G 200, 3. Elektrophorese. Aktive Fraktionen jeweils durch Ultrafiltration eingeengt, bei $-5^{\circ} \mathrm{C}$ aufbewahrt und vor Auftrag zentrifugiert (10 $\mathrm{min}$ $25000 \mathrm{rpm}$ ). Elektrophorese auf Sepha$\operatorname{dex} G 25$ als Träger in $0,02 \mathrm{~mol} / \mathrm{l}$ Natriumphosphatpuffer pH 6,5, Säule $2 \times 50 \mathrm{~cm}$. $22 \mathrm{~mA}, 420 \mathrm{~V}$, 50 Stunden, $\rightarrow \mathrm{K}^{-}$. o- 0 Protein 口- $\square$ Kathepsin
Der Erfolg der verschiedenen Separationsmethoden ist in Tabelle 1 dargestellt, wobei der Reinigungseffekt erfaßt wurde mit der Änderung des Proteingehaltes. Vier Kathepsinpräparate, die 3-7 Separierungsschritte $(\bar{x}=5)$ durchlaufen hatten, zeigten am Ende im Mittel eine spezifische Aktivität von $\bar{x}=163$; eine Ausbeute von $\bar{x}=12 \%$ und einen Reinigungseffekt von $\bar{x} 210$ fach Bei einigen Präparaten wurde ein höherer Reinigungseffekt bis zum 380 fachen erreicht.

Die Haltbarkeit der Enzympräparate ist in dèr folgenden Ubersicht dargestellt (Tab. 2).

\section{Eigenșchaften der Énzyme}

An den gereinigten Kathepsinpräparaten und den aktiven Fraktionen von Phosphoproteid-Phosphatase und Saurer Phosphatase, gewonnen auf Sephadex G 200, wurden folgende Meßergebnisse erzielt:

\section{Molekulargewicht}

Aus Sephadex-Gel G 100, geeicht mit 6 bekannten Proteinen, eluierte die Kathepsinfraktion mit dem Distri- butionskoeffizienten 0,312 , was sich fast völlig deckte mit Ovalbumin. Daraus ergibt sich ein Molekulargewicht von etwa 45000.

Die Bestimmung des Einstein Stokes Radius, die Prof. Felgenhauer (30) von der Universitäts-Nervenklinik Köln freundlichst für uns durchführte, ergab an einem auf Sephadex G 200, G 100 und G 75 gereinigten Präparat (Spezifische Aktivität 52,3) mit der PolyacrylamidgelElektrophorese 4 Banden, von denen die beiden mittleren die intensivsten waren, während die schnell wandernde nur in ganz geringer Menge vorhanden war. Es ergab sich für alle vier Fraktionen ein identischer Einstein-Stokes Radius von $2,9 \mathrm{~nm}$. Unterstellt man, daß es sich um globuläre Proteine handelt, entspräche das einem Molekulargewicht von 46000 .

Die Phosphoproteid-Phosphatase eluierte von Dextrangel G 200 mit dem Distributionskoeffizienten 0,198, was ein Molekulargewicht von etwa 180000 anzeigt.

Die Saure Phosphatase eluierte von Sephadex G $200 \mathrm{mit}$ dem Distributionskoeffizienten 0,819, was ein Molekulargewicht von etwa 23000 anzeigt. 
Tab. 1. Verteilung von Kathepsin aus der löslichen Fraktion aus Rinderhirn-Cortex unter aufeinanderfolgenden Reinigungsschritten. Konzentrierung mit gesättigter Ammoniumsulfatlösung.

\begin{tabular}{|c|c|c|c|c|c|c|}
\hline \multirow[b]{2}{*}{ Reinigungsschritt } & \multirow[b]{2}{*}{$\begin{array}{l}\text { Protein } \\
\text { [g] }\end{array}$} & \multicolumn{2}{|c|}{ Aktivität } & \multicolumn{2}{|c|}{ Spezif. Aktivität } & \multirow[b]{2}{*}{ Reinigung } \\
\hline & & $\begin{array}{l}\text { Tyrosin- } \\
\text { Äquivalente } \\
{[\mu \mathrm{mol} / \mathrm{h}]}\end{array}$ & $\begin{array}{l}\text { Tyrosin- } \\
\text { Âquivalente } \\
\text { [ } \mu \mathrm{mol} / \mathrm{min}]\end{array}$ & $\begin{array}{l}\text { Tyrosin- } \\
\text { Aquivalente } \\
\text { [nmol/min - } \\
\text { mg Protein] }\end{array}$ & $\begin{array}{l}\text { Ausbeute } \\
{[\%]}\end{array}$ & \\
\hline $\begin{array}{l}\text { Lösliche Fraktion } \\
\text { Ammoniumsulfatfällung } \\
\text { Sephadex G } 200 \\
\text { Sephadex G } 100 \\
\text { Elektrophorese } \\
\text { Sephadex G } 100\end{array}$ & $\begin{array}{l}4,870 \\
2,780 \\
0,261 \\
0,039 \\
0,019 \\
0,003\end{array}$ & $\begin{array}{r}274 \\
256 \\
240 \\
202 \\
98,8 \\
64,2\end{array}$ & $\begin{array}{l}4,57 \\
4,27 \\
4,0 \\
3,37 \\
1,65 \\
1,07\end{array}$ & $\begin{array}{c}0,94 \\
1,54 \\
15,3 \\
86,4 \\
86,9 \\
357\end{array}$ & $\begin{array}{l}93,4 \\
87,4 \\
73,7 \\
36 \\
23,4\end{array}$ & $\begin{array}{r}1,6 \\
16,2 \\
91,9 \\
92,4 \\
380\end{array}$ \\
\hline
\end{tabular}

Tab. 2. Haltbarkeit der Enzympräparate

\begin{tabular}{lll}
\hline & $\begin{array}{l}\text { Proteinkonz. } \\
{[\mathrm{g} / \mathrm{l}]}\end{array}$ & $\begin{array}{l}\tau 1 / 2 \\
{[\mathrm{~d}]}\end{array}$ \\
\hline Kathepsin & ca. $0,5-10$ & 20 \\
Phosphoproteid-Phosphatase & ca. $2-15$ & 30 \\
Saure Phosphatase & ca. 1,5 & 1,5 \\
& ca. 10 & 6 \\
\hline
\end{tabular}

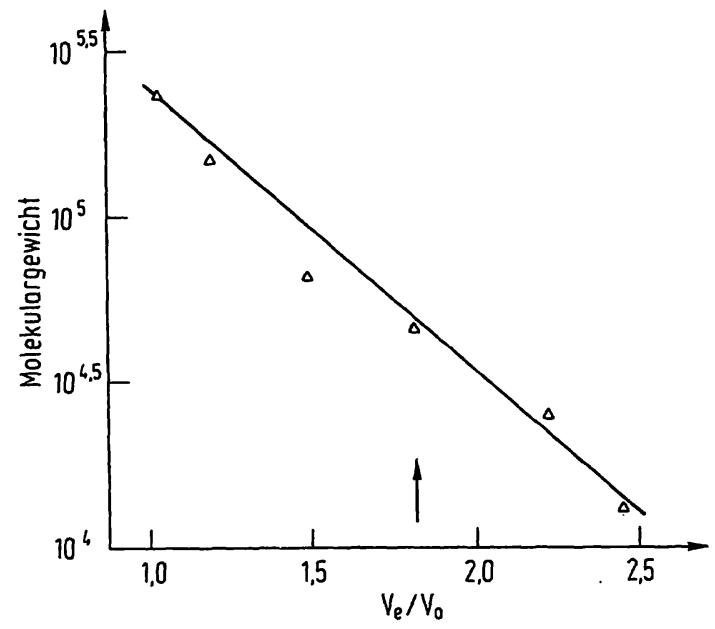

Abb. 5. Bestimmung des Molekulargewichtes von Kathepsin aus dem Elutionsvolumen nach Gelfiltration auf Sephadex G 100, Säule 3,2 $\times 220 \mathrm{~cm}$. Aufgetragene Proteine von bekanntem Molekulargewicht in der Reihenfolge ihrer Elutionsvolumina: Katalase, Aldolase, Rinderalbumin, Ovalbumin, Trypsinogen, Cytochrom C.

\section{Analytische Gel-Elektrophorese (Kathepsin)}

Ein stärker gereinigtes Kathepsinpräparat $(1 \times$ Sephadex G 200, 3 X G 100, 3 X Elektrophorese, spezifische Aktivität 162) wurde für uns von Prof. Rüdiger, Biochemisches Institut der Universität Köln, mittels analytischer Gel-Elektrophorese untersucht (Abb. 6). Es überwiegt eine scharfe Bande bei $2 \mathrm{~cm}$ Wanderungsstrecke über einem schmalen Untergrund schneller wandernder Proteine.

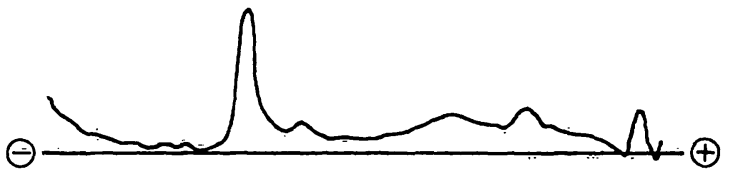

Abb. 6. Analytische Gelelektrophorese.

Kathepsinpräparat separiert auf Sephadex G 200, $3 \times$ G 100, $3 \times$ Elektrophorese, spezifische Aktivität (Tyrosinäquivalente) $162 \mathrm{nmol} / \mathrm{min} \cdot \mathrm{mg}$ Protein. Geprüft mit der analytischen Gelelektrophorese (Standardverfahren nach $R$. Maurer), $50 \mathrm{~mA}, 600 \mathrm{~V}, 55 \mathrm{~min}$. Anfärbung mit Amidoschwarz. Auswertung im Handauswertegerät von Bender und Hobein, München. Als Markierung der Wanderungsstrecke diente Bromphenolblau.

Bestimmung der Sedimentationskonstanten (Kațhepsin) Ein mehrfach gereinigtes Kathepsinpräparat $(1 \times$ Sepha$\operatorname{dex}$ G 200, 1 X G 100, 3 X Elektrophorese, spezifische Aktivität 109) wurde in der analytischen Ultrazentrifuge (Beckman Modell E) für uns von Herrn Prof. Rüdiger, Biochemisches Institut Köln, freundlichst untersucht. Es ergab sich eine Sedimentationskonstante von 3,9 \pm $0,13 \mathrm{~s}_{20, \mathrm{w}}$. (Wegen sehr geringer Eiweißkonzentration Fehler bis zu $20 \%$ möglich).

\section{Aminosäureanalyse (Kathèpsin)}

Zwei Kathepsinpräparate wurden nach der Methode von Spackman, Moore \& Stein (31) mit dem Spinco Modell 120 Aminosäure-Analysator untersucht durch Dr. Eaker, Biochemisches Institut Uppsala. 1. Kathepsinpräparat: $1 \times$ Sepadex G 200, 6 X Sephades G 100, Endausbeute 12\%. 2. Kathepșinpräparat: $1 \times$ Sephadex G 200, 3 X Sephadex G 100, 1 X G 75, 1 X Agar-Gél-Elektrophorese, $1 \times$ G 75, Endausbeute 7\%. Nach Säurehydrolyse zeigten sich beide Präparate aus den gleichen Aminosäuren aufgebaut mit Mengenunterschieden (Tab. 3).

Kinetik (Kathepsin, Phosphoproteid-Phosphatase und Saure Phosphatase)

pH-Optimum

Kathepsin wies ein breites pH-Optimum auf im schwach sauren Bereich mit einem Maximum der Aktivität um pH 5,7. Die Halbwertsbreite betrug 2,5 pH-Einheiten. 
Tab. 3. Aminosäure-Zusammensetzung von Kathepsin.

Die Werte in Klammern sind mittlere Literaturwerte, die mit Rücksicht auf die Ausgewogenheit der übrigen Restanteile eingesetzt wurden. Einzelheiten siehe Text.

\begin{tabular}{|c|c|c|c|c|c|c|}
\hline & $\begin{array}{l}\text { Präparat } 1 \\
\mu \mathrm{mol} / 0,63 \mathrm{mg} \text { ) }\end{array}$ & Reste/Mol & Mol \% & $\begin{array}{l}\text { Präparat } 2 \\
\mu \mathrm{mol} / 0,39 \mathrm{mg})\end{array}$ & Reste/Mol & Mol \% \\
\hline Tryptophan & $(0,1)$ & $(6,99)$ & - & $(0,1)$ & $(11,54)$ & - \\
\hline Lysin & 0,3775 & 26,4 & 6,57 & 0,2435 & 28,10 & 5,69 \\
\hline Histidin & 0,1028 & 7,2 & 1,79 & 0,0635 & 7,33 & 1,48 \\
\hline Ammoniak & $(0,3)$ & $(20,9)$ & - & 0,9840 & 113,57 & 23,0 \\
\hline Arginin & 0,2296 & 16,0 & 4,00 & 0,1359 & 15,68 & 3,18 \\
\hline Asparaginsäure & 0,6130 & 42,8 & 10,7 & 0,3267 & 37,71 & 7,64 \\
\hline Threonin & 0,2836 & 19,8 & 4,93 & 0,1711 & 19,75 & 4,00 \\
\hline Serin & 0,3879 & 27,1 & 6,75 & 0,3075 & 35,49 & 7,19 \\
\hline Glutaminsäure & 0,6703 & 46,8 & 11,7 & 0,3177 & 36,67 & 7,43 \\
\hline Prolin & 0,3252 & 22,7 & 5,7 & 0,1849 & 21,34 & 4,32 \\
\hline Glycin & 0,4699 & 32,8 & 8,2 & 0,3471 & 40,06 & 8,11 \\
\hline Alanin & 0,4238 & 29,6 & 7,4 & 0,2491 & 28,75 & 5,82 \\
\hline Halb-cystin & 0,0775 & 5,4 & 1,4 & $(0,1)$ & $(11,54)$ & - \\
\hline Valin & 0,4105 & 28,7 & 7,1 & 0,2117 & 24,43 & 4,95 \\
\hline Methionin & 0,1084 & 7,6 & 1,9 & 0,0546 & 6,3 & 1,28 \\
\hline Isoleucin & 0,2718 & 19,0 & 4,7 & 0,1571 & 18,1 & 3,67 \\
\hline Leucin & 0,5263 & 36,8 & 9,2 & 0,2808 & 32,41 & 6,56 \\
\hline Tyrosin & 0,2299 & 16,1 & 4,0 & 0,0954 & 11,01 & 2,23 \\
\hline Phenylalanin & 0,2578 & 18,0 & 4,5 & 0,1256 & 14,49 & 2,92 \\
\hline
\end{tabular}

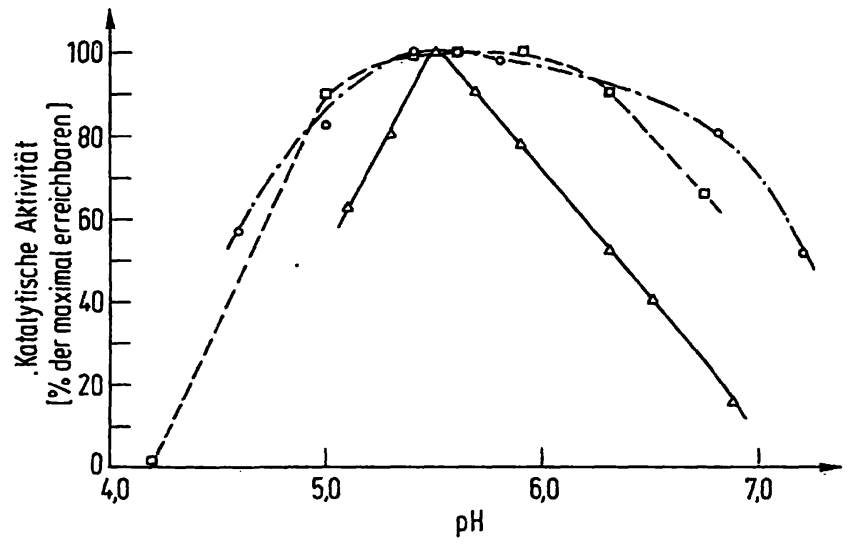

Abb. 7. pH-Öptimum

Kathepsinpräparat: spezifische Aktivität (T̄yrosinäquivalente) $52 \mathrm{nmol} / \mathrm{min} \cdot \mathrm{mg}$ Protein. Im Ansatz von $0,3 \mathrm{ml} 0,05 \mathrm{ml}$ Enzympräparat. Inkubiert $60 \mathrm{~min}$ bei $37^{\circ} \mathrm{C}$. Phosphat-Citrat-Ammoniak-Puffer nach Willstätter-Grassmann (37). Phosphoproteid-Phosphatase: Im Ansatz vọn 0,8 ml: 0,05 ml Enzympräparat (spezifische Aktivität (Phosphat) 2,61 nmol/min - mg Protein), Acetat-Veronalpuffer nach Michaeliș (35). Inkubiert $20 \mathrm{~min}, 38^{\circ} \mathrm{C}$. Saure Phosphatase: Im Ansatz von $0,8 \mathrm{ml}: 0,1 \mathrm{ml}$ Enzympräparat (1671 PhosphataseEinheiten/mg Protein), Acetat-Veronalpuffer nach Michaelis (35), inkubiert $60 \mathrm{~min}$ bei $38^{\circ} \mathrm{C}$. $100 \%$ Umsatz entsprechen: Kathepsin (Tyrosinäquivalente) $9,66 \mathrm{nmol} /$ min - ml. Phosphoprodeidphosphàtase (Phosphat)

$6,65 \mathrm{nmol} / \mathrm{min} \cdot \mathrm{ml}$ sowie Phosphatase 94 PhosphataseEinheiten $/ \mathrm{ml}$.

$\square--\rightarrow$ Kathepsin

$\Delta-\Delta$ Phosphoproteidphosphátáse

o-.-.-o Saurè Phosphatase.

Die Phosphoproteid-Phosphätase war durch ein schärferes pH-Optimum mit einem Maximum bei pH 5,5 ausgezeichnet. Die Halbwertsbreite betrug 1,3 pH-Einheiten. Die
Saure Phosphatase zeigte den breitesten pH-Wirkungsbereich mit einem Optimum bei pH 5,6 und einer Halbwertsbreite von 2,6 pH-Einheiten.

Optimale Inkubationstemperatur

Kathepsin hatte unter den 3 Enzymen die steilste Temperaturabhängigkeit mit Optimum bei $37^{\circ} \mathrm{C}$. Bei höheren Temperaturen fiel die Aktivität sehr schnell ab und war bei $50^{\circ} \mathrm{C}$ praktisch verschwunden.

Die Phosphoproteid-Phosphatase hatte ihr TemperaturOptimum bei $45^{\circ} \mathrm{C}$.

Die Saure Phosphatase hatte das höchste Temperaturoptimum bei $54^{\circ} \mathrm{C}$. Selbst bei $60^{\circ} \mathrm{C}$ waren beide Enzyme noch zu 50\% aktiv.

\section{Optimale Inkubationsdauer}

Die Aktivität verlief linear der Zeit für Kathepsin und Saure Phosphatase während $30 \mathrm{~min}$, für die Phosphoproteid-Phosphatașe während $20 \mathrm{~min}$.

\section{Optimale Substratkonzentration}

Bei Kathepsin stieg die Ënzymaktivität bis zur Substratkonzentration von $6 \mathrm{~g} / 1$ Casein Hammarsten an. Dann setzte eine Substratinhibition ein. Die Caseinkonzentration, bei der der Extinktionsunterschied (bei $280 \mathrm{~nm}$ ) die Hälfte des Maximalwertes erreichte, war 2,75 g/l. Für dia Phosphoproteid-Phosphatase war die optimale Substratkonzentration $10 \mathrm{~g} / 1$ Casein isoelectric, das entspricht $82 \mathrm{mg} / 1$ caseingebundenem Phosphor. Bei 1,43 g/l Casein war die halbe Maximalgeschwindigkeit erreicht. Darüber trat auch hier Substratinhibition ein. 


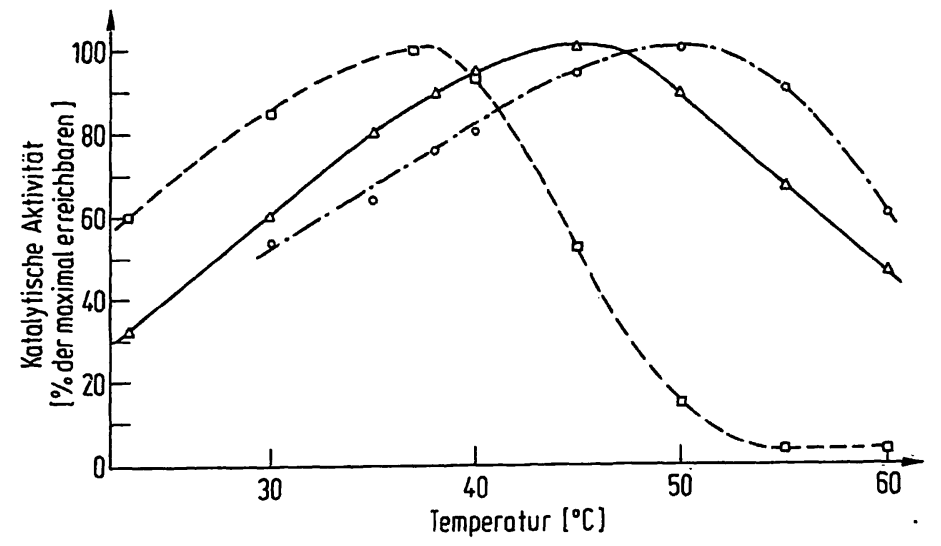

Abb. 8. Temperatur-Optimum.

Kathepsin: Im Ansatz von 0,3 ml : 0,05 ml Enzympräparat (Tyrosinäquivalente) $52,3 \mathrm{nmol} / \mathrm{min} \cdot \mathrm{mg}$ Protein. Inkubiert $30 \mathrm{~min}$ pH 5,4. PhosphoproteidPhosphatase: Im Ansatz von 0,8 $\mathrm{ml}: 0,05 \mathrm{ml}$ Enzympräparat (Phosphat) $2,61 \mathrm{nmol} / \mathrm{min} \cdot \mathrm{mg}$ Protein. Inkubiert $40 \mathrm{~min}, \mathrm{pH}$ 5,5. Saure Phosphatase: Im Ansatz von $0,8 \mathrm{ml}: 0,1 \mathrm{ml}$ des Enzympräparates (1671 Phosphatase-Einheiten/mg Protein), inkubiert 50 min pH 5,6. 100\% Umsatz entsprechen: Kathepsin (Tyrosinäquivalente) $14,6 \mathrm{nmol} / \mathrm{min} \cdot \mathrm{ml}$. Phosphoproteidphosphatase (Phosphat) $5,7 \mathrm{nmol} / \mathrm{min} \cdot \mathrm{ml}$. Saure Phosphatase 120 Phosphatase-Einheiten $/ \mathrm{ml}$.

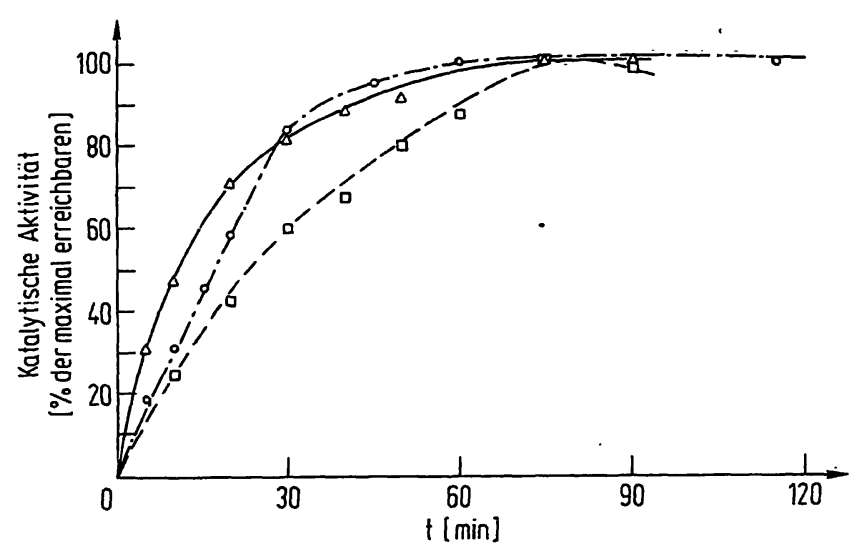

Abb. 9. Optimale Inkubationsdauer.

Kathepsinpräparat: Spezifische Aktivität (Tyrosinäquivalente) $109 \mathrm{nmol} / \mathrm{min} \cdot \mathrm{mg}$ Protein. Im Ansatz von $0,3 \mathrm{ml}: 0,1 \mathrm{ml}$ Enzympräparat, inkubiert bei pH 5,4 $37^{\circ} \mathrm{C}$. Phosphoproteid-Phosphatase: Im Arisatz von $0,8 \mathrm{ml}: 0,05 \mathrm{ml}$ Enzympräparat (Phosphat), $2,61 \mathrm{nmol} /$ $\mathrm{min} \cdot \mathrm{mg}$ Protein, inkubiert bei $\mathrm{pH} 5,5 / 45^{\circ} \mathrm{C}$. Saure Phosphatase: Im Ansatz von 0,8 $\mathrm{ml}: 0,1 \mathrm{ml}$ Enzympräparat (1671 Phosphatase-Einheiten/mg Protein). Inkubiert bei pH 5,6/50 ${ }^{\circ} \mathrm{C}$. $100 \%$ Umsatz entsprechen: Kathepsin (Tyrosinäquivalente) $8,2 \mathrm{nmol} / \mathrm{min} \cdot \mathrm{ml}$ Phosphoproteidphosphatase (Phosphat) 3,1 nmol/min . $\mathrm{ml}$. Saure Phosphatase 78 Phosphatase-Einheiten/ml. Zeichenerklärung wie Abb. 7.

Bei der Sauren Phosphatase trat keine Substrathemmung auf. Die $K_{\mathrm{m}}$ betrug für Na-Phenolphthaleindiphosphat $2 \mu \mathrm{mol} / \mathrm{l}$.

\section{Diskussion}

Aus der löslichen Fraktion aus Rinderhirn-Cortex wurden 3 Enzyme isoliert, abgrenzbar durch Unterschiede des Molekulargewichtes, der kinetischen Eigenschaften und der Wirkung auf das Substrat. Die Saure Phosphatase wurde hier nur im nicht-biologischen Sinne charakterisiert durch ein körperfremdes Substrat Na-Phenolphthaleindiphosphat. Aus anderem Zusammenhang weiß man aber, daß sie mit dem Proteinumsatz verknüpft ist. Von den beiden Proteinasen im Gehirn: Kathepsin und Phos-
phoproteid-Phosphatase ist bekannt, daß sie fast nur Phosphoproteide angreifen, hier gemeinsam an der Aufspaltung von Casein geprüft.

Das Kathepsin stellte sich auf Dextran-Gel als einzelne, einheitliche Fraktion dar. Allerdings ergab die Polyacrylamidgel-Elektrophorese eines noch nicht stärker gereinigten Präparates (spezifische Aktivität 52,3) 4 Banden, die alle den gleichen Molekülradius hatten. In einem stärker gereinigten Präparat (spezifische Aktivität 162) trat nur eine Hauptbande hervor über einem schmalen, eiweißhaltigen Untergrund. Daher liegt möglicherweise das Hirnkathepsin in Isoenzymen vor mit einer Hauptfraktion, die bei weitergehender Reinigung alleine zurückbleibt. In der Leber sind von Kathepsin D mehrere Isoenzyme beschrieben (1) und von Kathepsin B1 mindestens 6 Isoenzyme (10). Auch im Hirn der Ratte stellten sich saure und neutrale Proteinase als Gruppe von Enzymen dar, die aber wegen ähnlicher Eigenschaften aus Dextran-Gel jeweils als einzelner Gipfel eluierten (26).

Es wurde in immer neuen Separationen versucht, das Enzymprotein rein darżustellen. Dabei zeigte sich regelmäßig die Kathepsinaktivität asymmetrișch dem Proteingipfel aufgelagert. Nur nach physikalischer Einwirkung auf das Proteinmolekül durch Frieren und Tauen, überlange Ultrafiltration, wiederholtes Zentrifugieren, Schwenken und Rühren, vielleicht auch durch die Erniedrigung des $\mathrm{pH}$ nach Fällung mit festem Ammoniumsulfat, spaltete sich aus dem aktiven Proteinpräparat eine inaktive Eiweißfraktion ab, die sich unter Elektrophorese entgegengesetzt, zur Anode, bewegte. Gleichzeitig gewann die aktive Fraktion raschere Beweglichkeit zur Kathode, deckte sich fast symmetrisch mit dem zugeordneten Proteingipfel und eluierte von Dextrangel später, was eine Verkleinerung des Moleküls anzeigt. Es spielten also wahrscheinlich in dem Präparat noch Assoziations- und Dissoziationsvorgänge eine Rolle. Vielleicht wurde ein fest mit mit dem Enzym verbundenes Begleitprotein nach Einwirkụng gewisser physikalischer Faktoren abgespalten. Kathepsin könnte in der Zelle physiologisch fest mit einem Hemmprotein verbunden sein als wirksamer Schutz gegen Selbstverdauung. Dieses könnte dưrch 
wiederholte, leichte mechanische Einwirkungen abgespalten werden ohne Verschwinden, aber unter Schmälerung der Aktivität.

Aus dem Elutionsvolumen bei Gelfiltration ergab sich ein Molekulargewicht für Kathepsin von etwa 45000 . Das entspricht dem Molekulargewicht von Kathepsin D aus menschlicher Leber (1). Aus der analytischen Gel-Elektrophorese ergab sich ein Molekulargewicht von 46000 . Da mit Hilfe der Säulenchromatographie und der analytischen Gel-Elektrophorese identische Molekülgröße gefunden wurde, kann man annehmen, daß es sich um ein normal konfiguriertes, globuläres Proteinmolekül handelt.

Die Bestimmung der Sedimentationskonstanten von Kathepsin in der analytischen Ultrazentrifuge ergab den Wert von $3,9 s_{20, w} \pm 0,13$. Ähnliche Sedimentationskonstante und Molekulargewicht fanden sich bei anderen globulären Enzymproteinen mit Proteinase-Aktivität:

$\begin{array}{lll} & \text { s}_{20, w} & \text { Mol.Gew. } \\ \text { Kathepsin: Dorschleber } & 3,31 & 58000 \\ \text { Saure Proteinase: } \text { A spergillus } & 3.33 & 34900 \\ \text { Proteinase: B. thermoproteolyticus } & 3,6 & 37000 \\ \text { Pepsinogen } & 3.24 & 40000\end{array}$

Die Aminosäure-Zusammensetzung wurde zwar nur an bis 380 fach gereinigten Präparaten geprüft. Dennoch ergab sich der für hochgereinigte Proteinasen anderer Herkunft (Säuger-Pepsin, A spergillus-Proteinase $(17,28)$ ) typische Aufbau aus sauren Aminosäuren bei sehr niedrigem Gehalt an basischen und schwefelhaltigen Aminosäuren.

Enzymkinetisch fiel auf, dạß im Gegensatż zu der an anderen Proteinasen beobachteten Hitzestabilität (5)
Hirnkathepsin empfindlich ist gegen Temperaturen über $40^{\circ} \mathrm{C}$, wobei es rasch seine Aktivität verliert, was auch von Guroff (6) beobachtet wurde. Dagegen waren Phosphoproteid-Phosphatase und Saure Phosphatase weit unempfindlicher gegen Wärme.

Die in der Literatur für Proteinasen beschriebene Substrathemmung $(6,20)$ trat auch hier bei beiden Proteinasen hervor. Sie wird durch Chelatbildung mit aktivierenden Metallen oder Verunreinigungen erklärt (6).

Umgekehrt war eine Notwendigkeit zur Aktivierung durch Metalle oder andere Aktivatoren nicht zu erkennen.

Die pH-Optima der drei untersuchten, mit dem Proteinumsatz verknüpften Enzyme lagen dicht zusammen, wodurch gemeinsamer Angriff am Substrat erleichtert werden könnte.

Die in der Kälte aufbewahrten Präparate der 3 Enzyme, unkonzentriert oder eingeengt, hielten sich nicht unbegrenzt, sondern verloren allmählich ihre Aktivität, was für schleichende Selbstverdauung auch in der Kälte spricht.

Die rote Proteinfraktion ist im Eluat aus Sephadex-Gel stets der Vorderflanke der Kathepsinaktivität verhaftet, konnte aber unter Elektrophorese durch raschere Wanderung zur Kathode abgetrennt werden. Ihr Absorptionsmaximum liegt bei $420 \mathrm{~nm}$. Danach könnte es sich um ein Hämprotein handeln.

\section{Danksagung}

Herrn Professor K. Heinrich, Direktor der Psychiatrischen Klinik Düsseldorf, wird freundlichst gedankt fir zeitliche Freistellung zur Niederschrift dieser Arbeit.

\section{Literatur}

1. Barrett, A. J. (1970), Biochem. J. 117, 601-607.

2. Dingle, J. T., Barrett, A. J. \& Weston, P. D. (1971); Biochem. J. $123,1-13$.

3. Morrison, G. I. G., Barrett, A. J., Dingle, J. T. \& Prior, D. (1973), Biochim. Biophys. Acta 302, 411-419.

4. Schwabe, C. \& Sweeney, S. C. (1972), Biochim. Biophys. Acta $284,465-472$.

5. Haurowitz, F. (1957), Ann. New York Acad. Sci. 68, 3-10.

6. Guroff, G. (1964), J. Biol. Chem. 239, 149-155.

7. Suzuki, K. (1973), Vitamins (Japan) 47, 285-295. Ref. C. A. 79, (1973). 88667n.

8. Goettlich-Riemann, W., Young, J. O. \& Tappel, A. L. (1971), Biochim. Biophys. Acta 243, 137-146.

9. Liao Huang, F. \& Tappel, A. L. (1971), Biochim. Biophys. Acta 236, 739-748.

10. Barrett, A. J. (1973), Biochem. J. 131, 809-822.

11. Greenbaum, L. M. \& Fruton, S. J. (1957), J. Biol. Chem. 226, 173-180:

12. Lichtenstein, N. \& Fruton, J. S. (1960), Proc. Nat. Acad. Sci. U.S. 46, 787-79i.

13. Liao Huang, F. \& Tappel, A. L. (1972), Biochim. Biophys. Acta: $268,527--538$.
14. De Lumen, B. O. \& Tappel, A. L. (1973), Biochim. Biophys. Acta 293, 217-225.

15. Lieberherr, M., Vreven, J. \& Vaes, G. (1973), Biochim. Biophys. Acta 293, 160-169; 170-177.

16. Lenney, J. F. \& Dalbec, J. M. (1969), Arch. Biochem. Biophysics $129,407-409$.

17. Ottesen, M. \& Rickert, W. (1970), Comp. Rend. Trav. Lab. Carlsberg 37, 301-325.

18. Horiuchi, S., Yamasaki, M. \& Yamada, Y. (1969), Sci. Papers Coll. Gen. Educ. Univ. Tokyo 19, 141-150.

19. Hayashi, R., Oka, Y., Doi, E. \& Hata, T. (1968) a) Agric. Biol. Chem. (Tokyo) 32, 359-366.

b) Agric. Biol. Chem. (Tokyo) 32, 367-373.

20. Rose, S. P. R. \& Heald, P. J. (1961), Biochem. J. 81, 339347.

21. Rabinowitz, M. \& Lipmann, F. (1960), J. Biol. Chem. 235, 1043-1050.

22. Rodnight, R. (1966), in „Protides of the Biological Fluids.“ (Hrsg. H. Peeters) S. 39. Elsevier, Publ. Amsterdam.

23. Rose, S. P. R. (1962), Biochem. J. 83, 614-622.

24. Ansell, G. B. \& Richter, D. (1954), Biochim. Biophys. Acta $13,87-91$. 
25. Palladin, A. V., Polyakova, N. M. \& Lishko, V. K. (1963), J. Neurochem. 10, 187-194.

26. Marks, N. \& Lajtha, A. (1965), Biochem. J. 97, 74-83.

27. Beaufay, H., Berleur, A. M. \& Doyen, A. (1957), Biochem. J. $66,32 \mathrm{p}$.

28. Albert, E. (1955), Hoppe-Seyler's Z. Physiol. Chem. 302, 129-141.

29. Albert, E. (1968), diese Z. 6, 38-41.

30. Felgenhauer, K. (1971), Clin. Chim. Acta 32, 53-57.

31. Spackman, D. H., Stein, W. H. \& Moore, S. (1958), Anal. Chem. 30, 1190-1206.

32. Sober, H. A. Ed. (1970), Hdb. of Biochemistry , 2. Aufl. S.C-15, Chemical Rubber Co. Cleveland, Ohio.
33. Levin, O. (1962) in „Methods in Enzymology“ (Colowick, S. P. \& Kàplan, N. O. Ed) Bd. 5, p. 27, Academic Press, New York and Londọn.

34. Huggins, C. \& Talalay, P. (1945), J. Biol. Chem. 159, 399410.

35. Michaelis, L. (1931), Biochem. ì. 234, 139-141/Documenta Geigy Wiss. Tab. 6. Aufl. Basel S. 277.

36. Kuttner, Th. \& Lichtenstein, L. (1930), J. Biol Chem. 86, 671-676.

37. Duspiva, F. (1939), Protoplàsma 32, 211-229.

38. Lowry, O. H., Rosebrough, N. J., Farr, A. L. \& Randall, R. J. (1951), J. Biol. Chem. 193, 265-275.

Prof. Dr. E. Albert

Psychiatrische Klinik der Universität Düsseldorf

D-4000 Düsseldorf

Bergische Landstr. 2 